\title{
Onset of Convection in a Very Compressible Fluid : The Transient Toward Steady State
}

\author{
Horst Meyer and Andrei B. Kogan* \\ Department of Physics, Duke University, Durham, NC 27708-0305. \\ *present address: Department of Physics, MIT, Cambridge, MA 02139-430\%.
}

\begin{abstract}
We analyze the time profile $\Delta T(t)$ of the temperature difference, measured across a very compressible supercritical ${ }^{3} \mathrm{He}$ fluid layer in its convective state. The experiments were done along the critical isochore in a Rayleigh-Bénard cell after starting the vertical constant heat flow $q$. For $q$ sufficiently well above that needed for the convection onset, the transient $\Delta T(t)$ for a given $\epsilon \equiv\left(T-T_{c}\right) / T_{c}$, with $T_{c}=3.318 \mathrm{~K}$, shows a damped oscillatory profile with period $t_{o s c}$ modulating a smooth base profile. The smooth profile forms the exponential tail of the transient which tends to the steady-state $\Delta T(\infty)$ with a time constant $\tau_{\text {tail }}$. The scaled times $t_{o s c} / t_{D}$ and $\tau_{\text {tail }} / t_{D}$ from all the data could be collapsed onto two curves as a function of the Rayleigh number over $\sim 3.5$ decades. Here $t_{D}$ is the characteristic thermal diffusion time. Furthermore comparisons are made between measurements of a third characteristic time $t_{m}$ between the first peak and the first minimum in the $\Delta T(t)$ profile and its estimation by Onuki et al. Also comparisons are made between the observed oscillations and the $2 \mathrm{D}$ simulations by Onuki et al. and by Amiroudine and Zappoli. For $\epsilon<9 \times 10^{-3}$ the experiments show a crossover to a different transient regime. This new regime, which we briefly describe, is not understood at present.

PACS numbers: 44.25.+f, 464.70.Fx, 47.47.Te
\end{abstract}

\section{INTRODUCTION}

Many common fluids can be treated as incompressible, i.e. one can assume that their density, while perhaps varying slightly with temperature, is pressureindependent. This is one of the assumptions of the so called Boussinesq approximation[1]. Throughout this paper, we refer to a fluid as "compressible" if the nonzero value of the isothermal expansion coefficient $\beta_{T}=$ $1 / \rho(\partial \rho / \partial P)_{T}$ significantly influences the fluid dynamics and thus cannot be neglected. Here $\rho$ is the density and $P$ the pressure.

The increased interest in understanding the properties of a compressible flow is driven by both experimental and theoretical work on highly developed turbulence (See the review article by L.P. Kadanoff[2] and references therein). Compressible supercritical fluids allow to explore flow regimes which are not easily accessible experimentally: for example, Prandtl numbers $P r$ of the order of 500 can be obtained, while for an ordinary gas $P r$ is of order 1[1].

A standard experimental approach to study flow is to use a Rayleigh-Bénard (RB) geometry, i.e. a laterally confined horizontal layer of fluid filling the space between two horizontal surfaces [3-5]. In a typical experiment, one applies externally heat with flux density $q$, and measures the temperature difference $\Delta T(t)$ across the layer. The observable quantity of interest to theory is the steady state value $\Delta T(\infty)$ as a function of $q$. If the fluid properties are known, the relations $N u(R a, P r)$ can be obtained and compared with predictions. Here $N u$ and $R a$ are the Nusselt and Rayleigh numbers, which represent the normalized heat current and temperature difference, respectively.

Generally, a compressible fluid does not obey the
Boussinesq approximation. However, in a RayleighBénard geometry, the Boussinesq approximation is always valid provided that the layer height is sufficiently small given the value of $\beta_{T}$. And vice versa, even a weakly compressible fluid will violate the Boussinesq approximation if the layer thickness is large enough[6]. Therefore, while the effects due to finite $\beta_{T}$ are interesting on their own, they can also pose a challenge in understanding results of experiments focusing on other phenomena: for example, studies of highly turbulent flow are often performed in tall RB cells, which is desirable because of the rapid $\left(\sim h^{3}\right)$ increase of the Rayleigh number with the cell height.

We are interested in the effects due to non-vanishing $\beta_{T}$, and we use supercritical ${ }^{3} \mathrm{He}$ as a test fluid. Supercritical fluids provide a unique opportunity to study the compressibility-related effects, because $\beta_{T}$ exibits a rapid divergence at the critical point. As a result, one can continuously change the system from a weakly-compressible state to a regime where the dynamics is dominated by the high compressibility. The analysis presented here extends that presented in an earlier paper [7] and focuses on the time-dependent transient $\Delta T(t)$. As explained there in detail, the apparatus design was chosen so that the high compressibility is the only significant deviation from the set of assumptions required by the Boussinesq approximation.

Even in the absence of convection, e.g. under zero gravity conditions, the dynamics of a highly compressible fluid can be qualitatively different from an incompressible system. As was first predicted theoretically by Onuki and Ferrell [8], and then later demonstrated experimentally by several groups[9], the density-temperature dynamics following a small disturbance of the tempera- 
ture of the walls depends on whether the pressure $P$ or the total volume $\mathrm{V}$ ( in other words, the space-average density $\langle\rho\rangle$ ) is kept constant. In the latter case, the mechanism called "Piston Effect" dominates the dynamics: Following a small positive step in the wall temperature, sharp gradients of $\rho$ and $T$ form quickly at the walls and produce "boundary layers", which expand away from the walls into the bulk of the fluid. The "piston" time scale for the formation of the boundary layer is typically seconds, while the relaxation back to the uniform density distribution happen on the diffusive time scale which in practice is minutes to hours. These boundary layers compress the bulk fluid adiabatically, which gives rise to an apparent fast relaxation of the bulk temperature: it becomes correlated with the wall temperature on the piston time scale instead of that of the diffusion. At constant $P$, or if the compressibility is small, the density field does not respond to the wall temperature variations, no boundary layers are formed and the temperature undergoes a conventional diffusive relaxation according to the Fourier Law.

If the fluid is subjected to the earth gravity field $g$, the most basic compressibility- related effect is the change in the conditions for the mechanical stability. For a supercritical fluid, the compressibility increases as $\left(T-T_{c}\right)^{-\gamma_{e f f}}$ with $\gamma_{e f f} \approx 1.19$ for ${ }^{3} \mathrm{He}$, as the critical point is approached from above along the critical isochore. As a result, the onset transition to the convective state exhibits a crossover from the usual Rayleigh- to the Schwarzschild criterion. Far above $T_{c}$, the temperature drop across the fluid layer of height $L$ at the convection onset transition is given by $\Delta T_{\text {ons }}=\Delta T_{R a}$ while close to $T_{c}$, the stability regime is determined by the "adiabatic temperature gradient" $\left.\nabla T\right|_{a d}[1]$ so that $\Delta T_{a d}=$ $\left.\nabla T\right|_{a d} \times L$. Here $\Delta T_{R a}$, the onset temperature for the Rayleigh criterion alone and $\left.\nabla T\right|_{a d}$ are given by

$$
\Delta T_{R a}=R a_{c}\left(D_{T} \eta / g \rho \alpha_{P} L^{3}\right)
$$

and

$$
\left.\nabla T\right|_{a d} \equiv\left(\frac{\partial T}{\partial p}\right)_{s} \frac{d p}{d z}=\rho g T \alpha_{P} / C_{P}=\rho g\left(1-\frac{C_{V}}{C_{P}}\right)\left(\frac{\partial T}{\partial p}\right)_{\rho} .
$$

Here $\rho, \mathrm{g}, \eta, \alpha_{P}, D_{T}$ and $C_{P}$ are respectively the density, gravitational acceleration, shear viscosity, coefficient of isobaric thermal expansion, thermal diffusivity and isobaric specific heat[10]. For cells with large aspect ratio, the theoretical value of the critical Rayleigh number $R a_{c}$ is $\approx 1708$. In general, as has been shown previously, $[11,12]$

$$
\Delta T_{\text {ons }}=\Delta T_{a d}+\Delta T_{R a}
$$

A "potential temperature" $\theta$ can be defined as

$$
\frac{\partial \theta}{\partial z}=\frac{\partial T}{\partial z}-\left.\nabla T\right|_{a d}
$$

where $z$ is the vertical coordinate (See for instance ref.[1]). When $\theta$ is substituted in place of $T$ into the hydrodynamic equations for a compressible fluid, the Boussinesq form is recovered, provided the large $\left.\nabla T\right|_{a d}$ causes the only deviation from the Boussinesq set of criteria (A,B,C and D in Appendix to Chapter 14 in ref[1]). This substitution leads to the corrected forms $N u^{\text {corr }}$ and $R a^{\text {corr }}$ where account is taken for the effect of compressibility. Here

$$
R a^{\text {corr }}=\frac{R a\left(\Delta T-\Delta T_{a d}\right)}{\Delta T}, N u^{\text {corr }}=\frac{\left(\Delta T_{\text {diff }}-\Delta T_{a d}\right)}{\left(\Delta T-\Delta T_{a d}\right)}
$$

with $R a \equiv \alpha_{P} \rho g L^{3} \Delta T / \eta D_{T}$. Also $\Delta T_{\text {diff }}$ is the temperature drop across the fluid in the diffusive regime for the same heat current $q$ that produces the observed $\Delta T$. For a discussion of these corrected numbers, we refer to ref[7].

Very recently, we reported a study of the convection in supercritical ${ }^{3}$ He. [7]. The experiments were carried out in a Rayleigh-Bénard cell with a layer thickness $L=0.106$ $\mathrm{cm}$ and aspect ratio of 57 . The top boundary was kept at a constant temperature. A constant heating power was applied at the bottom plate, the temperature of which was left floating. Both the top and the bottom surfaces had a high thermal conductivity and thus there was no lateral temperature inhomogeneity. A temperature difference $\Delta T(t)$ was measured across the fluid layer as a function of time $t$ after the heat current was started. Its value in the steady state is henceforth denoted as $\Delta T$ or as $\Delta T(\infty)$.

The cell was filled to the critical density $\rho=\rho_{c}$ and then sealed, thus the experiments were always done under fixed volume conditions. The reduced temperature $\epsilon=\left(T-T_{c}\right) / T_{c}$, where $T_{c}=3.318 \mathrm{~K}$ is the critical temperature, was varied over the range $0.2 \geq \epsilon \geq 5 \times 10^{-4}$, where the fluid properties changed substantially, for instance $1.2 \times 10^{-6}<\beta_{T}<1.5 \times 10^{-3} \mathrm{~cm}^{2} /$ dyne and $2<\operatorname{Pr}<590$. The effects of gravity on the vertical density distribution were at most of the order of a few $\%$ at the temperatures closest to $T_{c}$. The heat currents $q$ across the fluid were kept small enough that there were no significant changes of the fluid properties across the fluid layer, even those strongly dependent on $\epsilon$ and on $\rho$. The fluid system was therefore never highly non-linear in the dependence of properties on $z$ in the cell. In addition, as mentioned earlier, all the assumptions required for the validity of the Boussinesq approximation, except the compressibility requirements, were fulfilled. For the numerical estimates of the maximum deviations as well as the experimental procedures and details, see ref [7].

Over the range $0.009 \leq \epsilon \leq 0.2$, patterns with damped oscillations were observed. Their frequency $t_{\text {osc }}^{-1}$ increased with $q$ and eventually the oscillations were smoothed out (Fig.9 of ref.[7]). For $\epsilon \leq 0.009$, the $\Delta T(t)$ profiles changed appreciably from those just described. These unusual transients stimulated theoretical studies of this supercritical fluid system, undertaken independently by Amiroudine and Zappoli (AZ) $[13,14]$ and by Onuki and coworkers $[15,16]$. Both groups carried out simulation 
studies with different codes and approximations, and some of their respective results will be discussed in this paper in connection with our data analysis. The simulations used the Navier Stokes equation and the heat conduction equation where a term was added in both, which takes into account the compressibility as represented by the adiabatic temperature gradient. The simulations were in 2D, and in ref.[15] the Stokes approximation was used, where in the N-S equation the time derivative of the velocity was set to zero. The fluid was contained in a cell with an aspect ratio of 4 with vertical periodic boundary conditions. In ref. [16], the Stokes approximation was not used and the simulations then extended to $R a^{\text {corr }}-R a_{c}=4 \times 10^{6}$. In the work by AZ[14], the simulations were carried out up to $R a^{c o r r}-R a_{c}=6 \times 10^{6}$ with the use of a supercomputer. Here various aspect ratios were used, but always with periodic boundary conditions. Furthermore the instability onset time of the same fluid, after the heat current start, was theoretically investigated as a function of $q$ and $\epsilon$ by Carlès[17] and by El Khoury and Carlès[18].

To describe a transient, we introduce several characteristic time scales that can be extracted from recorded data. Two of these, the period of damped oscillations $t_{\text {osc }}$ and the relaxation time $\tau_{\text {tail }}$ of the transient tail end, give in scaled form a particularly interesting universal result, which has not been predicted so far. In Section II, we give an overview of various types of profiles $\Delta T(t)$, and the conditions under which they are observed, and we define the characteristic times used in the analysis. This is followed in Section III by the scaled presentation of $t_{o s c}$ and $\tau_{\text {tail }}$ versus the Rayleigh number. In Section IV, another measured characteristic time in the $\Delta T(t)$ profile, $t_{m}$ as defined by Chiwata and Onuki[15], is compared with their predictions and simulations. Also the simulation results by AZ are discussed. The temperature region for $\epsilon<9 \times 10^{-3}$, where no oscillations were observed, is briefly described. Finally Section V presents the main results and conclusions.

\section{CONVECTION ONSET AND TRANSIENT PROFILES}

We first give an overview of the various profiles $\Delta T(t)$ observed after the start of the constant heat flow $q$ at $t$ $=0$, and we find that they exhibit several distinct characteristic shapes. For each of these, one can identify an approximate range in the parameters $\epsilon$ and $\Delta T(\infty)$, or a "zone" where the shape is observed. We first describe the typical $\Delta T(t)$ shapes and the corresponding "zones" in the $\Delta T(\infty)-\epsilon$ plane. Then, we define two characteristic times and discuss their respective uncertainties. Because the $\Delta T(t)$ observed above and below $\epsilon \approx 9 \times 10^{-3}$ are quite different, we consider them separately.

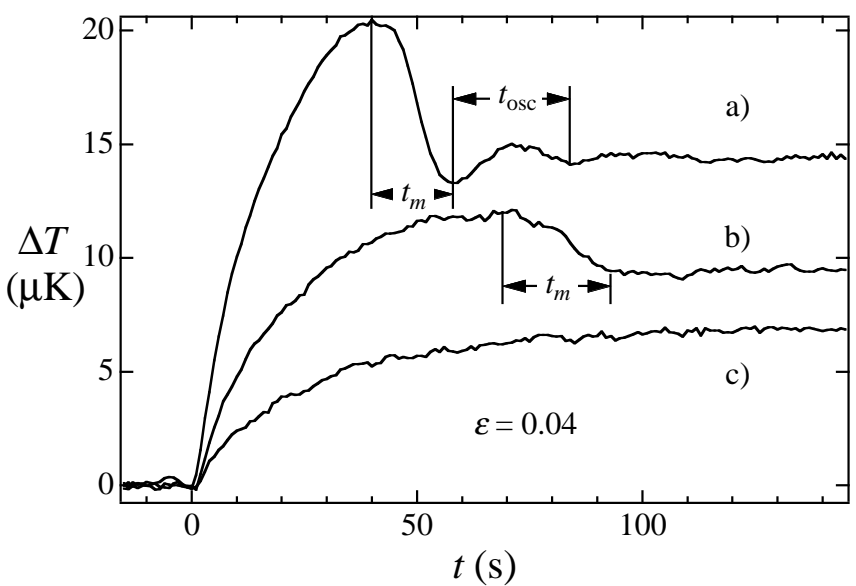

FIG. 1. Recordings $\Delta T(t)$ after starting the heat current, with the definition of the times $t_{o s c}$ (Section III) and $t_{m}$ (Section IV). The recordings were made at $\epsilon=0.04$ with the heat flow $q$ (in $10^{-8} \mathrm{~W} / \mathrm{cm}^{2}$ a) $\left.5.17 \mathrm{~b}\right) 2.53$, c) 1.21 .

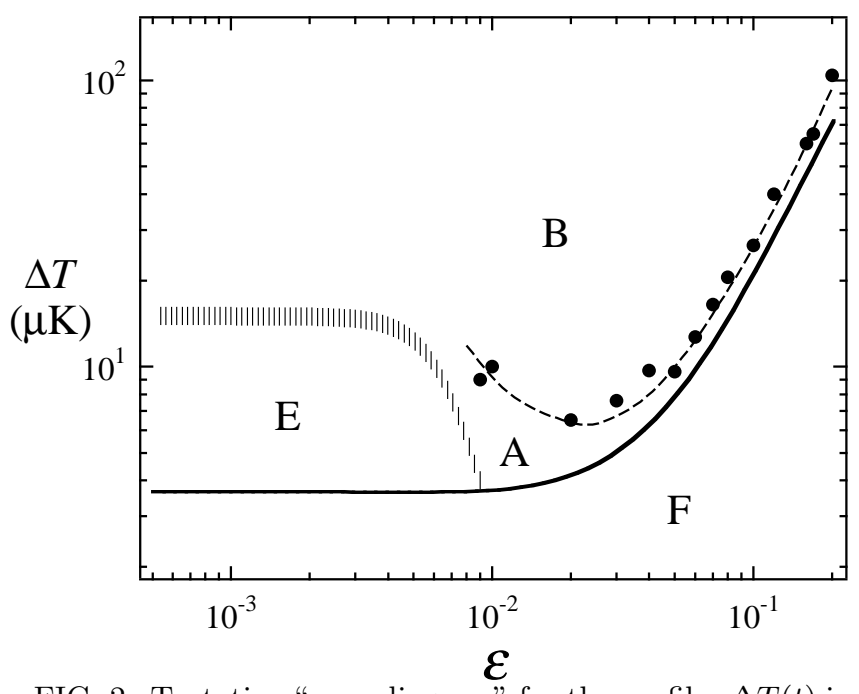

FIG. 2. Tentative "zone diagram" for the profiles $\Delta T(t)$ in the $\Delta T-\epsilon$ plane. The line of solid circles forms the boundary between zone $\mathrm{A}$ and $\mathrm{B}$. There are no data for this boundary below $\epsilon<9 \times 10^{-3}$, where no oscillations are observed. In zone $\mathrm{E}$, no overshoot is observed.

\section{A. Region for $\epsilon \geq 9 \times 10^{-3}$}

In the non-convective regime, the transient $\Delta T(t)$ follows the prediction of the Fourier Law, modified to accomodate the adiabatic - or "Piston" effect. An example trace can be seen in Fig.1c. $\Delta T(t)$ tends exponentially to $\Delta T(\infty)$ with a relaxation time determined by the thermal diffusivity $D_{T}$ [19]. This corresponds to zone $F$ of the diagram in Fig.2. The solid line separating zone $F$ from the rest of the field is given by the condition of the mechanical stability (Eq. 3) confirmed experimentally in [7].

When $q$ is increased above the value that corresponds to the convection onset, the transient $\Delta T(t)$ exibits an 
initial rise followed by an overshoot. This is a well-known effect which can be understood as a result of the fluid inertia $[20,21]$ : the convection doesn't start immediately after the mechanical stability condition is violated. Instead, $\Delta T(t)$ rises in accordance with the non-convective dynamics past the convection onset threshold until the fluid flow develops. Two examples of such behavior are shown at very different values of $\epsilon$ in Fig.1b and Fig.3b. This behavior is observed in zone A of Fig.2. Interestingly, we do not generally find the relaxation towards the $\Delta T(\infty)$ to be exponential, as predicted and experimentally observed in the immediate vicinity of the onset for Boussinesq fluids $[20,21]$. Instead, we find an almost linear decrease followed by a kink in the transient. The noise in Fig.1 obscures the "kink" which is clearly seen in Fig. $3 \mathrm{~b}$ at the end of the overshoot. We henceforth refer to the transients of this type as a "truncated oscillation".

A further increase in $q$ produces damped oscillations in $\Delta T(t)$ with a period $t_{o s c}$ (Figs 1a and 3a). Most of the analysis presented in this work focuses on this regime. These oscillations are observed in the lower part of zone B in Fig. 2 above its boundary with zone A. For $\epsilon<9 \times 10^{-3}$, this boundary is undetermined because of lack of data.

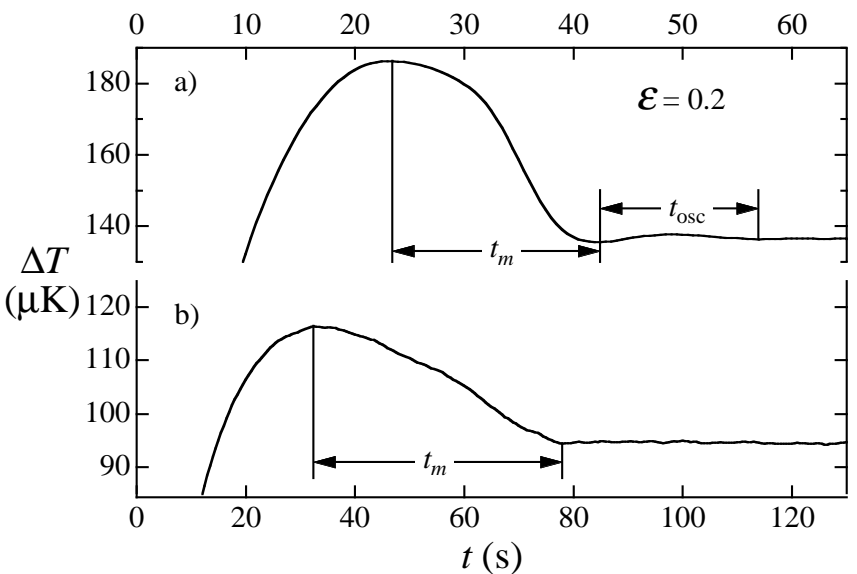

FIG. 3. Recordings $\Delta T(t)$ at $\epsilon=0.2$, with the definition of the times $t_{m}$ and $t_{\text {osc }}$. a) In zone B of fig.2 with damped oscillations and b) in zone A with truncated oscillations. The heat flow $q$ (in $10^{-7} \mathrm{~W} / \mathrm{cm}^{2}$ ) is a) 3.89 , b) 2.16 .

With increasing $q$ in zone $\mathrm{B}$, the profile $\Delta T(t)$ becomes more complex but has two components amenable to analysis: damped oscillations of period $t_{o s c}(q, \epsilon)$ followed by a smooth base profile with a minimum, beyond which $\Delta T(t)$ tends exponentially to $\Delta T(\infty)$ from below with a relaxation time $\tau_{\text {tail }}(q, \epsilon)$. This evolution with $q$ at $\epsilon=$ 0.05 can be seen in Fig.4, where for emphasis only the top portion of the $\Delta T(t)$ profile is shown. The visibility and relative amplitude of these components are functions of $\epsilon$ and of $q$. For a given $\epsilon$, both $\tau_{\text {tail }}$ and $t_{\text {osc }}$ decrease with increasing $q$, and always $t_{\text {osc }}<\tau_{\text {tail }}$. When $t_{\text {osc }}$ becomes comparable to the time constant of the thermometer circuitry, $\simeq 1.3 \mathrm{~s}$., the oscillation amplitude and eventually the very first peak become smoothed out and only the base profile relaxation is observed. Hence in a portion of the recorded traces, either $t_{o s c}$ or $\tau_{\text {tail }}$ can be determined, but not both.

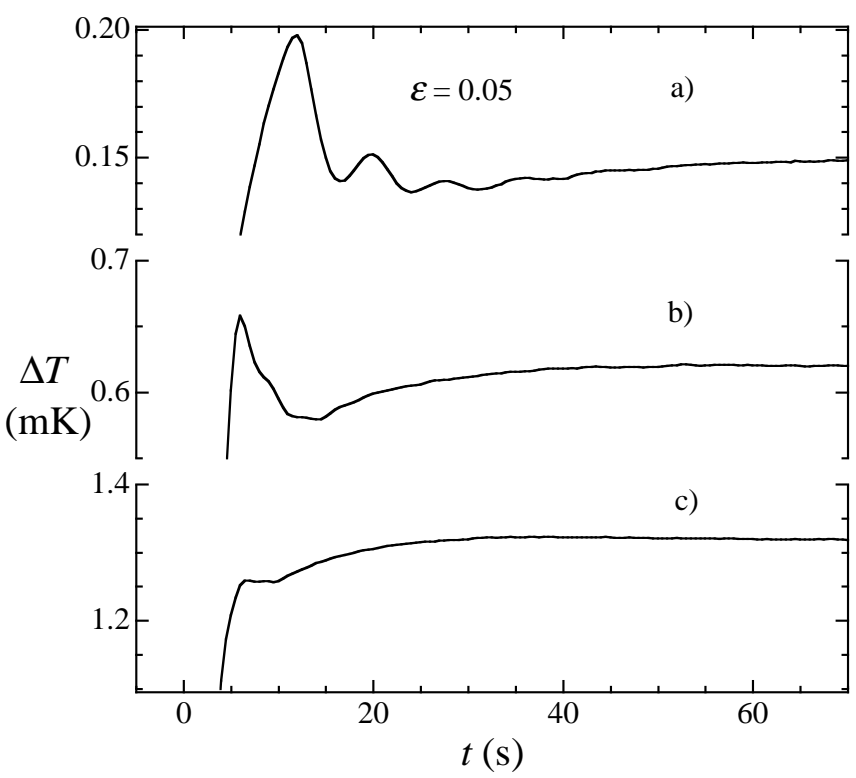

FIG. 4. Evolution of the profiles $\Delta T(t)$ at $\epsilon=0.05$ with increasing values of $q$ (in $10^{-7} \mathrm{~W} / \mathrm{cm}^{2}$ ) : a) 9.65 , b) 61.3 , c) 154 .

For the determination of $\tau_{\text {tail }}$, which ranged from $\sim$ 5 to 100 s., the recorded curve was fitted to an exponential, and a check of the dependence on the fit range showed an internal consistency in $\tau_{\text {tail }}$ within about \pm 10 $\%$. The relaxation profile distortion by the instrumental time constant has been considered [22]. Besides an effective overall lag, there is no significant change produced in the value of $\tau_{\text {tail }}$. The oscillation period $t_{\text {osc }}$ was obtained from the time count between succeeding minima, with an average error of approximately $\pm 10 \%$. In this determination, the position of the first maximum in $\Delta T(t)$ was not included. This was because the time $t_{m}$ between this maximum and the first minimum, as defined in ref.[15], is larger than half of the oscillation period $t_{o s c}$. This asymmetry is most evident for $\epsilon=0.2$, (See Fig.3a) and gradually decreases with $\epsilon$.

At high enough values of $q$, an additional very weak and broad maximum is observed before $\Delta T(\infty)$ is reached from above. The amplitude of this component is only at most $0.3 \%$ of $\Delta T(\infty)$ and the corresponding relaxation time to steady state is estimated to be about $\mathrm{O}\left(5 \tau_{\text {tail }}\right)$. In our analysis, we have neglected this additional component.

\section{B. Region for $\epsilon \leq 9 \times 10^{-3}$}

As already described in ref. [7], the profiles of $\Delta T(t)$ in this region are quite different from those at higher $\epsilon$. In zone $\mathrm{E}$ of Fig. 2, no overshoot is observed. After 
the start of the heat flow, the increase of $\Delta T(t)$ is found to be consistent with diffusive heat transfer[19] until the onset of instability leads to saturation and to the plateau $\Delta T(\infty)$, as shown by the three lowest traces in Fig.12a) of ref.[7]. Above the passage from zone $\mathrm{E}$ to $\mathrm{B}$, indicated by a hatched band in Fig.2, there is a gradual appearance of an overshoot with increasing $q$, but without oscillations.

\section{SCALED TIMES VERSUS RAYLEIGH NUMBER}

Steady state measurements in both compressible and essentially incompressible liquids [3-5] show approximate scaling of the data on a $N u^{\text {corr }}\left(R a^{\text {corr }}\right)$ plot leading to a universal curve. This is also the case for supercritical ${ }^{3} \mathrm{He}$ at various temperatures, but reported over a larger range of $\beta_{T}$ and $\operatorname{Pr}$ than previously. The degree of scaling of steady-state data can be seen in Fig.7a and 8 of ref.[7] (See however note[23]). Is there similarly for the transient data a corresponding representation of scaled times that leads to a universal curve? This section deals with this question.

Since data extend from the regime of early turbulence $\left(R a \sim 5 \times 10^{6}\right)$ to close to the convection onset, we express them in terms of the "distance" from this transition, $\left[R a^{\operatorname{corr}}-R a_{c}\right]$. It is useful here to present the relation with the measured quantities $\Delta T$ and $\Delta T_{\text {ons }}$. From a combination of Eqs. 3 and 5, one obtains

$$
\left[R a^{\text {corr }}-R a_{c}\right]=B(\epsilon)\left[\Delta T-\Delta T_{\text {ons }}\right]
$$

where $B(\epsilon) \equiv \rho g \alpha_{P} L^{3} /\left(\eta D_{T}\right)$, with numerical values showing a strong dependence on $\epsilon$ along the critical isochore, as presented in Table 1.

\begin{tabular}{r|c|c|c|c|c|c}
\hline \hline$\epsilon$ & $\beta_{T}\left(\mathrm{~cm}^{2} /\right.$ dyne $)$ & $\mathrm{D}\left(\mathrm{cm}^{2} / \mathrm{s}\right)$ & $\gamma$ & $\operatorname{Pr}$ & $B[\epsilon]\left(\mathrm{K}^{-1}\right)$ & $t_{D}(\mathrm{~s})$ \\
\hline \hline $5.0 \mathrm{e}-4$ & $1.52 \mathrm{e}-3$ & $7.3 \mathrm{e}-7$ & $3.0 \mathrm{e} 3$ & 590 & $7.0 \mathrm{e} 12$ & $3.8 \mathrm{e} 3$ \\
$1.2 \mathrm{e}-3$ & $5.7 \mathrm{e}-4$ & $1.4 \mathrm{e}-6$ & $1.2 \mathrm{e} 3$ & 290 & $1.4 \mathrm{e} 12$ & $2.0 \mathrm{e} 3$ \\
$2.0 \mathrm{e}-3$ & $2.91 \mathrm{e}-4$ & $2.3 \mathrm{e}-6$ & $6.6 \mathrm{e} 2$ & 180 & $4.3 \mathrm{e} 11$ & $1.2 \mathrm{e} 3$ \\
$5.0 \mathrm{e}-3$ & $9.61 \mathrm{e}-5$ & $5.5 \mathrm{e}-6$ & $2.5 \mathrm{e} 2$ & 75 & $6.1 \mathrm{e} 10$ & $5.1 \mathrm{e} 2$ \\
$1.0 \mathrm{e}-2$ & $4.30 \mathrm{e}-5$ & $1.1 \mathrm{e}-5$ & $1.2 \mathrm{e} 2$ & 38 & $1.4 \mathrm{e} 10$ & $2.6 \mathrm{e} 2$ \\
$2.0 \mathrm{e}-2$ & $1.88 \mathrm{e}-5$ & $2.2 \mathrm{e}-5$ & 58 & 19 & $3.1 \mathrm{e} 09$ & $1.3 \mathrm{e} 2$ \\
$5.0 \mathrm{e}-2$ & $6.32 \mathrm{e}-6$ & $5.4 \mathrm{e}-5$ & 23 & 7.4 & $4.2 \mathrm{e} 08$ & 52 \\
$1.0 \mathrm{e}-1$ & $2.77 \mathrm{e}-6$ & $1.1 \mathrm{e}-4$ & 12 & 3.8 & $9.8 \mathrm{e} 07$ & 27 \\
$2.0 \mathrm{e}-1$ & $1.21 \mathrm{e}-6$ & $1.9 \mathrm{e}-4$ & 6.5 & 2.1 & $2.4 \mathrm{e} 07$ & 15 \\
\hline \hline
\end{tabular}

TABLE I. Several properties (numbers rounded off) for ${ }^{3} \mathrm{He}$ along its critical isochore, relevant to the convection onset transient. $B[\epsilon]$, defined in Eq. 6 and $t_{D}=L^{2} / 4 D$ are functions of the layer thickness $L$ and have been calculated for the present cell, $L=0.106 \mathrm{~cm}$.
We have found that both $t_{\text {osc }}$ and $\tau_{\text {tail }}$, when scaled by the diffusive time $t_{D}=L^{2} / 4 D_{T}$ [24], and plotted versus $\left[R a^{\text {corr }}-R a_{c}\right]$, can be collapsed on two separate curves. The range of scaling extends over about 3.5 decades in $\left[R a^{\text {corr }}-R a_{c}\right]$. In Fig. 5 we show this scaling plot where the various symbols indicate the temperatures $\epsilon$ at which the transients were recorded[25]. There are some systematic deviations and scatter of less than $\pm 15 \%$ from the average curves.

This is a remarkable result because with decreasing $\epsilon$ from 0.2 to $9 \times 10^{-3}$, the isothermal compressibility $\beta_{T}$ and $t_{D}$ increase by factors of $\approx 40$ and 20 respectively, and the Prandtl number $\mathrm{Pr}$ increases from 2 to 42 . Over approximately 2.5 decades of $\left[R a^{\text {corr }}-R a_{c}\right]$, the curves for $t_{o s c} / t_{D}$ and $\tau_{\text {tail }} / t_{D}$ can be represented by power laws with exponents of $\simeq-0.52$ and -0.60 , as obtained from a "best" straight line through the points. Because of the non uniform data distribution over the range of Rayleigh numbers, a least-square fit seems inappropriate. Both scaled times diverge as $\left[R a^{\text {corr }}-R a_{c}\right] \rightarrow 0$ and there appears to be no clear dependence on $\mathrm{Pr}$, as shown by the collapsing of the data for various values of $\epsilon$. We can therefore expect that the representations in Fig. 5 should be the same for other fluids kept at constant volume, and hence they form in a crude first approximation a universal set of curves. We note that for convection transients observed at constant pressure in liquid ${ }^{4} \mathrm{He}[21], \Delta T(t)$ relaxes from its "overshoot" maximum towards $\Delta T(\infty)$ with a characteristic time that diverges as $\left[R a-R a_{c}\right] \rightarrow$ 0 , similarly showing the "slowing down" as the transition to fluid stability is approached.

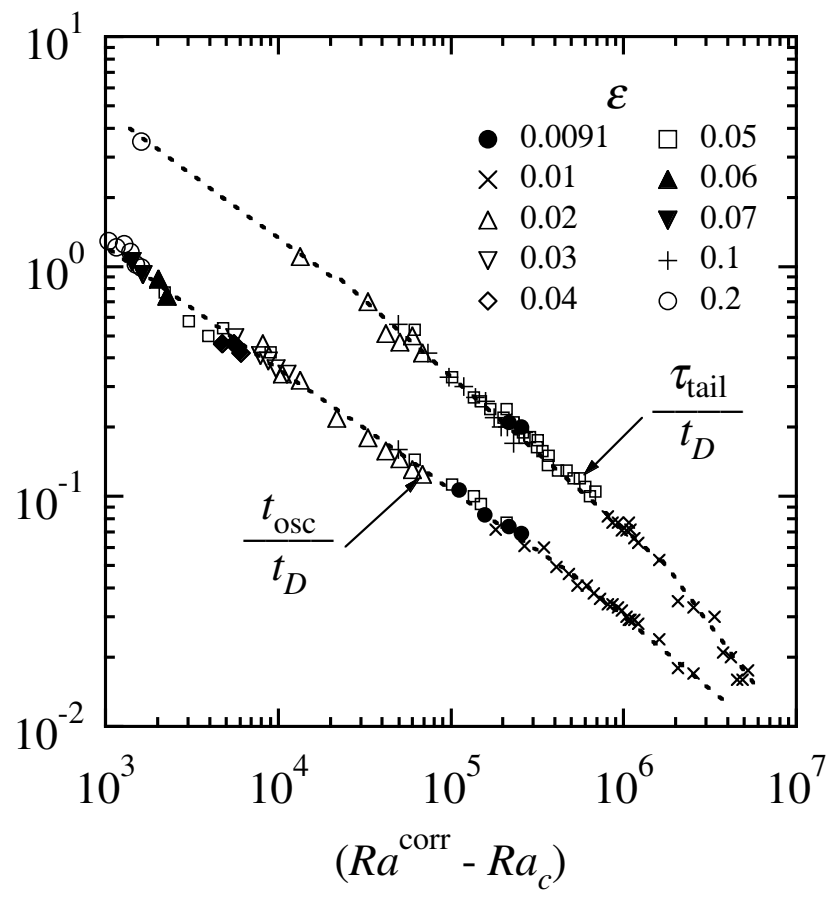

FIG. 5. Scaled plots of $t_{o s c} / t_{D}$ and $\tau_{\text {tail }} / t_{D}$ versus $\left[R a^{\text {corr }}-R a_{c}\right]$ for reduced temperatures $9 \times 10^{-3} \leq \epsilon \leq 0.2$. The dashed lines are "guides to the eye". 
As mentioned before, no damped oscillations were detected for $\epsilon<9 \times 10^{-3}$. To check whether this absence could be caused by the instrumental time constant if $t_{\text {osc }}$ becomes too short, a calculation of the expected $t_{\text {osc }}$ was made based on the scaling plot of Fig.5. This calculation was done for $\epsilon=5 \times 10^{-3}$ and $3 \times 10^{-3}$ and for several values of $\Delta T$ where experiments had been carried out. For these $\Delta T(t)$ profiles, the predicted $t_{\text {osc }}$ values were of order $15 \mathrm{~s}$., large enough to be clearly observable. Hence we conclude that the character of the transient has changed when $\epsilon$ is below $9 \times 10^{-3}$. Furthermore the $\tau_{\text {tail }} / t_{D}$ obtained from analysis of the $\Delta T(t)$ recordings at $\epsilon=5 \times 10^{-4}$ were found to be inconsistent with an extrapolation from the scaled plot in Fig.5 to the higher $R a$ numbers $\left(\sim 4 \times 10^{8}\right)$ corresponding to these data. The steady-state data at this temperature also showed an anomalous behavior in the $N u^{\text {corr }}\left(R a^{\text {corr }}\right)$ plot[7].

It has been shown in several papers [14-16] from simulations that the compressible fluids should give oscillatory $\Delta T(t)$ arbitrarily close to the convection onset. This behavior is associated with the formation of the boundary layers responsible for the "Piston Effect" and thus should occur in compressible fluids kept at constant volume, but not at constant pressure. The boundary layers are predicted to undergo an instability which leads to a sequence of "plumes" of hot and cold fluid moving across the cell periodically, and producing oscillations in $\Delta T(t)$. Eventually the oscillations disappear as the "plumes" give way to the time-independent convection rolls.

\section{COMPARISON OF EXPERIMENT WITH PREDICTIONS}

In this section we are concerned with the time $t_{m}$ between the first peak and the first minimum of the $\Delta T(t)$ profile (See Figs 1 and 3). Specific predictions for $t_{m}$ have been made analytically [15] as well as obtained from numerical simulations $[15,16]$. We extend the definition of $t_{m}$ to include the region of truncated oscillation, as shown in Fig $1 \mathrm{~b}$ and $3 \mathrm{~b}$, where the minimum has been replaced by a kink followed by a plateau. This truncated overshoot is found in Zone A of Fig.2. When plotted versus $\left[\Delta T-\Delta T_{\text {ons }}\right]$ or $\left[R a^{\text {corr }}-R a_{c}\right]$, the line of the $t_{m}$ data points for a given $\epsilon$ is continued smoothly from zone $\mathrm{B}$ into zone A. Here we note that in neither the simulations of ref[15] nor those of ref[14] is an evidence of the "truncated oscillations" as was observed experimentally (zone A). In the simulations, damped oscillations were obtained with a diverging period as $\left[\Delta T-\Delta T_{\text {ons }}\right] \rightarrow 0$.

\section{A. comparison with numerical simulations}

In Fig.6 we show the time $t_{m}$ as a function of $[\Delta T-$ $\left.\Delta T_{\text {ons }}\right]$, and the comparison between the experiments and the simulations[15] at $\epsilon=0.05$ for a range of heat currents $q$. As per Table $1,\left[\Delta T-\Delta T_{\text {ons }}\right]=2 \times 10^{-5}$ corresponds to $\left[R a^{\text {corr }}-R a_{c}\right]=8.5 \times 10^{3}$. It can be seen that both sets of points give almost parallel lines, with

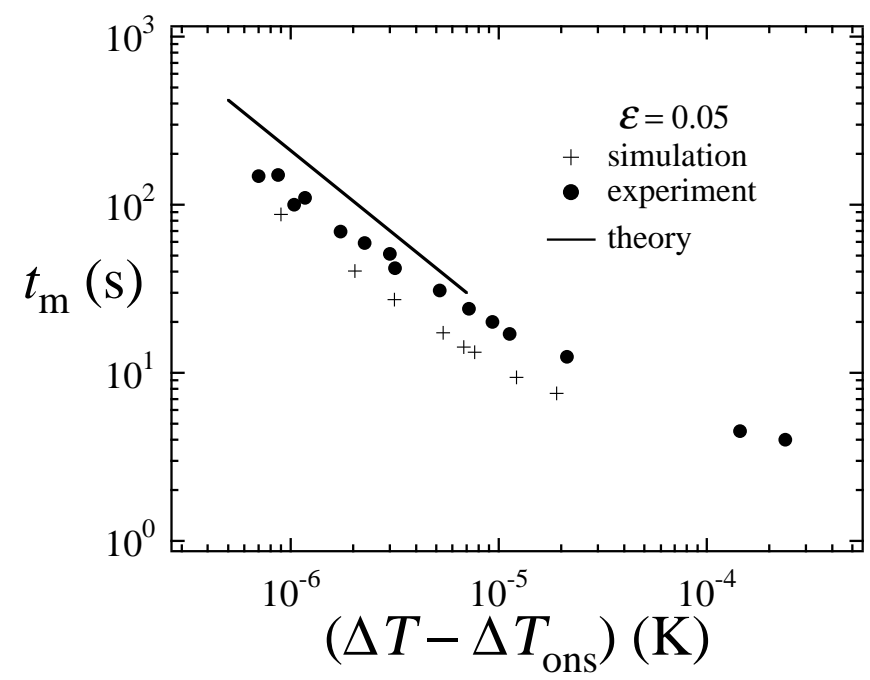

FIG. 6. Logarithmic presentation of $t_{m}$ versus [ $\Delta T-\Delta T_{\text {ons }}$ ] at $\epsilon=0.05$, from experiments and from simulations by Chiwata and Onuki [15]. The solid line represents the predicted behavior in the limit of small $\left[\Delta T-\Delta T_{\text {ons }}\right]$, Eq. 8 .

the amplitude difference being of the order of $30 \%$. In the limit of small heat currents (or small values of $[\Delta T-$ $\left.\Delta T_{\text {ons }}\right]$ ), the exponent of both lines of points tends to ($1)$. At high values of $\left[\Delta T-\Delta T_{\text {ons }}\right]$ or of $\left[R a^{\text {corr }}-R a_{c}\right]$ the exponent is found to be $\approx 0.5$, and this result is similar to that found in Fig.5 for the scaled $t_{o s c} / t_{D}$ plot.

A simple prediction for the value of the limiting slope can be obtained in the Stokes approximation, when $\left[R a^{c o r r} / R a_{c}-1\right] \leq \operatorname{Pr}(\epsilon)$ [15]. In this case, $t_{m}$ is estimated by setting $t_{m} \approx L / v$, the time it will take for the cooler fluid near the top of the cell to flow to the lower boundary. Here $v$ is the velocity estimated from arguments given under Eq.7 in ref.[15], and $t_{m}$ is then given by

$$
\left(t_{m} / t_{D}\right)\left[R a^{\text {corr }} / R a_{c}-1\right]=O(1)
$$

and, after combining with Eq.6, by

$$
t_{m}=O(1) t_{D} B(\epsilon)^{-1}\left[\Delta T-\Delta T_{\text {ons }}\right]^{-1}
$$

with $\mathrm{O}(1)$ a numerical factor of the order of 1 . (In ref.[15] the " $=\mathrm{O}(1)$ " was written as " $\approx$ ". See also $\operatorname{ref}[16]$, paragraph under Eq.3.8). This estimation, where we have set $\mathrm{O}(1)=1$ is shown by a solid line in Fig. 6 for $\epsilon=0.05$. The agreement between the estimations, the experiments and the simulations is within the expected uncertainty of the estimation.

The simulations have given impressive agreement with the experimental results under the steady-state conditions, for instance in the plot of $\Delta T$ versus $q$, (Fig.4 in ref[15]), in the convection current versus $R a^{\text {corr }}$, Fig. 4 
of ref[16] and also in the plots of $\Delta T(t)$ at $\epsilon=0.01$ by AZ[14]. While on the whole the agreement between simulations and experiments for the oscillatory transient is good, we note some minor inconsistencies. From the simulation plots, Figs.1 and 2 in ref.[15], Fig. 1a in ref.[16], both at 0.05 , and similarly from plots by AZ at $\epsilon=0.01$, the ratio $t_{o s c} / 2 t_{m}$ is always $>1$. By contrast, in the experiments the ratio is $<1$, as can be seen from Figs. 1a, $3 \mathrm{a}$ and $4 \mathrm{a}$ in this paper. Also in the simulations of ref [16], Fig.1a shows no evidence of the observed slow relaxation to the steady-state value of $\Delta T$. Here the simulations are compared with the same experimental data as in Fig.4a of the present paper. Hence, so far simulations do not provide a prediction for $\tau_{\text {tail }}$. Also, as mentioned before, they do not show truncated oscillations (zone A in Fig.2).

\section{B. Comparison with theory}

We have first examined for the range $0.009 \leq \epsilon \leq 0.2$ a plot of $t_{m} / t_{D}$ versus $\left[R a^{\text {corr }}-R a_{c}\right]$ similar to Fig. 5 . The $t_{m}$ data include results from both zones $\mathrm{A}$ and $\mathrm{B}$, and extend to a lower range of $\left[R a^{c o r r}-R a_{c}\right]$ than in Fig.5. However, in contrast to the $t_{o s c} / t_{D}$ plot, we found that the data did not fall on a single curve. The spread is particularly large at low values of $\left[R a^{\text {corr }}-R a_{c}\right]$. Hence such a scaled representation was not found informative. Instead, for the comparison of the prediction with experiments, it was found more convenient to formulate $t_{m}$ in terms of temperature differences $\left[\Delta T-\Delta T_{\text {ons }}\right]$ than of $\left[R a^{c o r r}-R a_{c}\right]$, as it gives a more compact data representation.

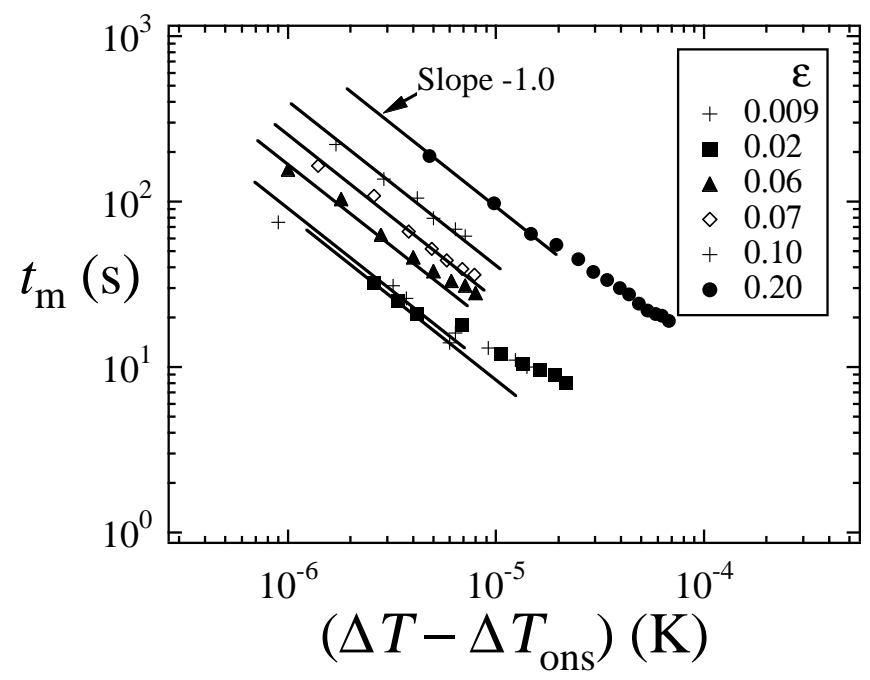

FIG. 7. Experimental data of $t_{m}$ versus $\left[\Delta T-\Delta T_{\text {ons }}\right]$ for selected reduced temperatures in the range $9 \times 10^{-3} \leq \epsilon \leq 0.2$. To avoid overcrowding, half of the curves at constant $\epsilon$ have been suppressed. The solid lines, all with slope of $(-1)$ are the result of a fit to the data in the limiting region of small values of $\left[\Delta T-\Delta T_{\text {ons }}\right]$. The slope $(-1)$ of the solid line represents the predicted behavior.
In Fig.7 we show experimental data sets at selected values of $\epsilon$, to avoid overcrowding. (The set for $\epsilon=0.05$, shown in Fig.6, has not been included here) and all were fitted with straight lines of slope of $(-1)$ in the limit of small values of $\left[\Delta T-\Delta T_{\text {ons }}\right]$. These fits gave the corresponding amplitudes, which were converted into amplitudes of $\left[R a^{\text {corr }}-R a_{c}\right]$ by means of Eq. 6. The result is presented in Fig.8 in the scaled form of Eq. 7 as a function of $\epsilon$. Since the expected ratio is of order unity [15], there is agreement with predictions at the higher values of $\epsilon$, but a systematic deviation appears as $\epsilon$ decreases below 0.02 , perhaps anticipating the crossover to the regime $\epsilon \leq 9 \times 10^{-3}$, where damped oscillations are no longer observed.

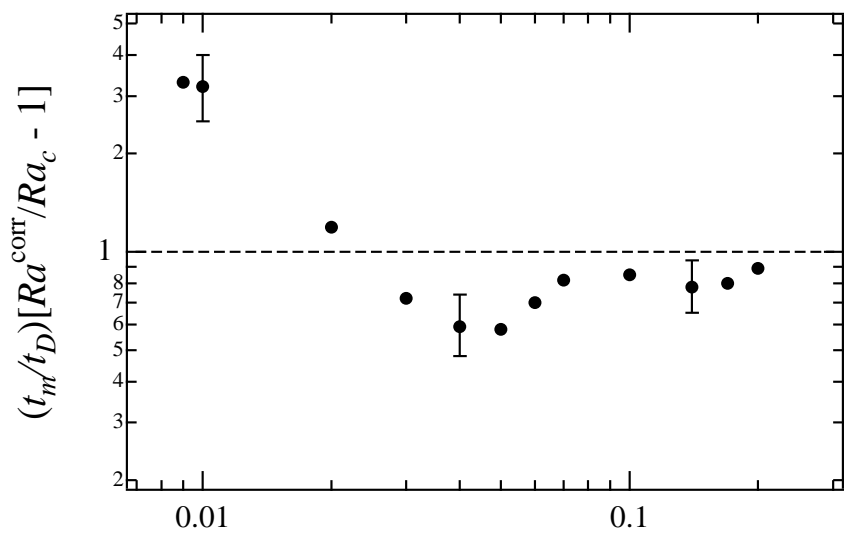

FIG. 8. The ratio $\left(t_{m} / t_{D}\right)\left[R a^{\text {corr }} / R a_{c}-1\right]$ from Eq.7 versus the reduced temperature $\epsilon$. The symbols (solid circles) represent the results obtained from the plot in Fig. 6 and 7 including all the values of $\epsilon$ where convection data were taken. According to the predictions[15], the ratio should be of the order of 1 and independent of $\epsilon$.

\section{DISCUSSION AND SUMMARY}

We have analyzed the time-dependent temperature difference $\Delta T(t)$ across the fluid layer along the critical isochore in a Rayleigh-Bénard cell with a height of $L=$ $0.1 \mathrm{~cm}$ and a large aspect ratio. The experiments studied the behavior of the fluid in its convective state from the start of a constant heat flow and until steady state was observed. The measurements were done with supercritical ${ }^{3} \mathrm{He}$ along the critical isochore where the physical properties change rapidly with the distance from $T_{c}$. A characteristic time for this system is $t_{D}=L^{2} / 4 D$, the diffusive time, where $D_{T}$ is the thermal diffusivity. The experiments have shown profiles of $\Delta T(t)$ gradually evolving with reduced temperature $\epsilon$ and heat flow $q$. The principal results can be summarised as follows.

1) The transient profiles appear to fall into two temperature regimes: above and below $\epsilon=9 \times 10^{-3}$. In the regime above, damped oscillations are observed, but not so in the regime below. The oscillations have subse- 
quently been reproduced in simulations by Onuki and his group and by Amiroudine and Zappoli at $\epsilon=0.05$ and 0.01 .

2) For $\epsilon \geq 9 \times 10^{-3}$, and for increasing heat flows, there is first a region of truncated oscillations. For larger heats, damped oscillations with a period $t_{o s c}$ are observed, and at still larger heat also a tail of the transient which tends to the steady-state value $\Delta T$ with a relaxation time $\tau_{\text {tail }}$. It is found that the scaled values of $t_{o s c} / t_{D}$ and $\tau_{\text {tail }} / t_{D}$ fall on two separate curves of points when plotted versus the Rayleigh number. This is remarkable, considering that the physical properties change very rapidly along the critical isochore.

3) For low Rayleigh numbers, predictions were made by Onuki and coworkers for the time $t_{m}$ between the first maximum and the following minimun in the oscillatory transient $\Delta T(t)$. We find their results to be in approximate agreement with our data.

4) While there is an impressive semiquantitative agreement between the observed $\Delta T(t)$ profiles, and those simulated in $2 \mathrm{D}$ under identical conditions of $L$ and fluid properties, there are some differences in detail. This might be due to the nature of the simulations where the geometry is different from that of the experimental cell. First, the simulations did not reveal a regime of truncated oscillations, as did the experiments. Second, there are subtle, but nevertheless visible differences in the oscillation patterns as shown by the ratios $t_{o s c} / 2 t_{m}$ which are $>1$ in the simulations but generally $<1$ in the experiments.

5) For $\epsilon<9 \times 10^{-3}$ no truncated or damped oscillations were detected while an extrapolation of the observations above this regime indicates that oscillations should have been observable. Hence the character of the profile $\Delta T(t)$ appears to be different in this temperature regime, and needs to be understood.

\section{ACKNOWLEDGMENTS}

One of us (HM) greatly acknowledges stimulating discussions and correspondence with A. Onuki, S. Amiroudine and P. Carlès. We thank A. Onuki for communicating the simulation data used in Fig.6. The efficient and willing help of F. Zhong and M. Gehm in formatting the figures is greatly appreciated. We also thank K. Sreenivasan and J. Niemela for their very appropriate suggested improvements in the text. This work has been supported by NASA grant No NAG3-1838.

[1] D.J. Tritton, Physical Fluid Dynamics (Oxford Science, Oxford, 1988) Chapter 14.

[2] L.P. Kadanoff, Physics Today 54, \# 8, 34 (2001).
[3] Sh. Ashkenazi and V. Steinberg, Phys. Rev. Lett 83, 3641 (1999).

[4] J.J. Niemela, L. Skrbek, K.R. Sreenivasan and R.J. Donnelly, Nature 404, 837 (2000).

[5] X. Chavanne, F. Chilla, B. Chabaud, B. Castaing and B. Hebral, Phys. Fluids 13, 1300 (2001).

[6] L.D. Landau and E.M. Lifshitz, Course of Theoretical Physics: Vol.6 Fluid Mechanics (Pergamon, Oxford, 1959)

[7] A.B. Kogan and H. Meyer, Phys. Rev. E 63 , 056310 (2001).

[8] A. Onuki and R.A. Ferrell, Physica A 64, 245 (1990).

[9] P. Guenoun, B. Khalil, D. Beysens, Y. Garrabos, F. Kammoun, B. Le Neindre and B. Zappoli, Phys. Rev. E 47,1531 (1993); H. Boukari, J.N. Shaumeyer, M.E. Briggs amd R.W. Gammon, Phys. Rev. A 41, 2260 (1990); H. Klein, G. Schmitz and D. Woermann, Phys. Rev. A 43, 4562 (1991); F. Zhong and H. Meyer, Phys. Rev. E 51, 3223 (1995); J. Straub, L. Eicher and A. Haupt, Intern. J. Thermophysics 16, 1033 (1995); A. Kogan and H. Meyer, J. Low Temp. Phys. 112, 419 (1998).

[10] For a simple meaning of $\left.\nabla T\right|_{a d}$ as $T_{c}$ is approached and $C_{P} / C_{V} \gg 1$, see ref.[7] below Eq. 2. Under these conditions, $\left.\nabla T\right|_{\text {ad }}$ tends to the constant value of $34 \mu \mathrm{K} / \mathrm{cm}$ for ${ }^{3} \mathrm{He}$.

[11] M. Gitterman and V. Steinberg, J. Appl. Math. Mech. USSR 34, 305 (1971). M. Gitterman, Rev. Mod. Phys. 50, 85 (1978).

[12] P. Carlès and B. Ugurtas, Physica D, 126, 69 (1999).

[13] S. Amiroudine, A.B. Kogan, H. Meyer and B. Zappoli, Proc. Internat. Congress Theor\& Appl. Mechanics, (ICTAM) Chicago Aug. 27, 2000, edited by H. Aref and J. W. Phillips, Kluwer Academic Publishers (2001).

[14] S. Amiroudine and B. Zappoli (private communication, and to be published).

[15] Y. Chiwata and A. Onuki, Phys. Rev. Lett. 87, 144301 (2001).

[16] A. Furukawa and A. Onuki Phys. Rev. E 66, 016302 (2002).

[17] P. Carlès, Physica D 147, 36 (2000).

[18] L. El Khoury and P. Carlès, (to be published).

[19] R.P. Behringer, A. Onuki and H. Meyer, J. Low Temp. Phys. 81, 71 (1990).

[20] R.P. Behringer and G. Ahlers, Phys. Lett. A 62, 329 (1977).

[21] R.P. Behringer, Rev. Mod. Phys. 57, 657 (1985).

[22] We are indebted to D. Schaeffer of the Duke U. Mathematics Department for producing an equation expressing the observed $\Delta T(t)$ signal in terms of an input signal modified by an instrumental time constant shorter than $\tau_{\text {tail }}$.

[23] The anomalous behavior observed at the highest values of $R a^{\text {corr }}$ is not understood and will not considered further here.

[24] This diffusion time is as defined in ref.[15], and differs from the usual diffusive time $L^{2} / D_{T}$ in ordinary fluids by a factor of 4 . This is because $t_{D}$ is chosen for the situation of a supercritical fluid where $C_{P}>>C_{V}$, and for relaxation at constant density. Under such conditions, the relaxation time is 4 times shorter than when $C_{P} \approx$ 
$C_{V}$ ( See ref.[19])

[25] A tabulation of scaled values of $t_{\text {osc }}$ and $\tau_{\text {tail }}$ is available from one of us (HM) for the temperature range $9 \times 10^{-3} \leq$ $\epsilon \leq 0.2$. Here the relevant values of $t_{D}$ are given for each $\epsilon$. 